2014-10

New best equivocation codes for syndrome coding

\author{
Al-Hassan, S
}

http://hdl.handle.net/10026.1/13722

10.1109/ictc. 2014.6983251

2014 International Conference on Information and Communication Technology Convergence (ICTC)

IEEE

All content in PEARL is protected by copyright law. Author manuscripts are made available in accordance with publisher policies. Please cite only the published version using the details provided on the item record or document. In the absence of an open licence (e.g. Creative Commons), permissions for further reuse of content should be sought from the publisher or author. 
See discussions, stats, and author profiles for this publication at: https://www.researchgate.net/publication/270880531

\section{New best equivocation codes for syndrome coding}

Conference Paper · October 2014

DOI: 10.1109/ICTC.2014.6983251

CITATIONS

3

3 authors, including:

Salah al-hassan

University of Plymouth

5 PUBLICATIONS 9 CITATIONS

SEE PROFILE

Some of the authors of this publication are also working on these related projects:

A Novel Induction Heating System View project

Project An adaptable interleaved DC-DC boost converter View project
READS

82

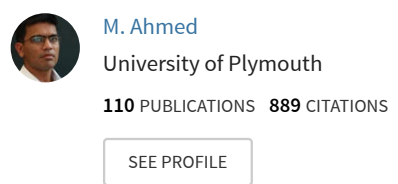




\title{
New Best Equivocation Codes for Syndrome Coding
}

\author{
Salah Al-Hassan, Mohammed Zaki Ahmed and Martin Tomlinson \\ School of Computing and Mathematics \\ University of Plymouth \\ United Kingdom \\ Email: salah.al-hassan, M.Ahmed, M.Tomlinson@plymouth.ac.uk
}

\begin{abstract}
In this paper we present a code design technique which produces codes for syndrome coding which have better secrecy than the best error correcting codes. Code examples are given for the case where the number of parity bits of the code is equal to 15. The code design technique presented is based on extensions of the parity check matrix of a set of good equivocation codes of shorter length. It is also shown that syndrome coding can be implemented without the traditional syndrome look up table, enabling any length codes to be used. An efficient recursive method to calculate the equivocation rate for the binary symmetric channel (BSC) and any linear binary code is also presented. The design results show that the best equivocation codes (BEC) that are produced have better equivocation rate for the syndrome coding scheme compared to all previously published codes, including the best known codes (BKC).
\end{abstract}

\section{INTRODUCTION}

The wiretap channel was proposed by Wyner [1], and is a physical layer model that takes the security of transmitted information into account. In this model, Alice (transmitter), wishes to send a secret message $M$ to Bob (legitimate receiver) in the presence of an eavesdropper (Eve). The wiretap channel model is shown in Fig. 1, where the main channel (between Alice and Bob) is an error-free channel and the eavesdropper channel is a Binary Symmetric Channel (BSC) with a probability of error $\left(p_{e}\right)$ [2]. Secrecy is measured by the equivocation rate.

Wyner showed that the equivocation rate approaches unity to the eavesdropper if codes are used that have length extending to infinity, if the syndrome space is chosen to be smaller than the Shannon entropy of the binary symmetric channel (BSC). Chen and Vinck [3] also investigated the binary symmetric wiretap channel, and they showed that the secrecy capacity can be obtained by using random linear codes with syndrome coding.

The syndrome coding scheme, whose basic idea is to convey information in the syndromes of a code so as to increase the communication security has been studied by several researchers. For example, Rouayheb and Soljanin [4] showed that network security can be achieved by using syndrome coding as an additional layer to a network code. Al-Hassan, Ahmed and Tomlinson [5] showed that the equivocation rate can be maximised on the eavesdropper side by using a combination of the technique of the McEliece cryptosystem using Best Known Codes (BKC) coupled with syndrome coding. Cohen and Zemor [6] analysed the information leakage of syndrome coding for the wiretap channel and proposed a

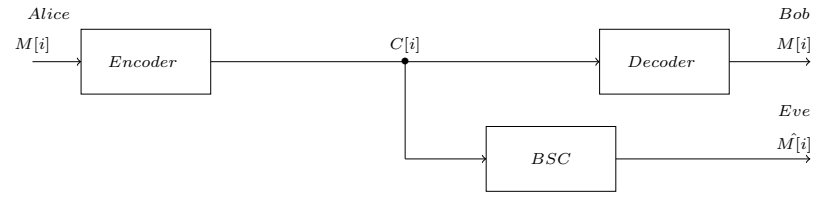

Fig. 1: Wiretap channel Model

method to select a syndrome function in order to minimise both the length of the transmitted vector and the information leakage to the eavesdropper. Code design for error correcting codes is an important and long standing topic in coding theory. Good codes can be designed by extending the parity check matrix of good codes as shown in [7], [8].

In this paper, we present an efficient recursive method for evaluating the equivocation rate of any linear, binary code when used in syndrome coding for the Binary Symmetric Channel (BSC). In addition, we present a code design technique to extend the binary linear $[n, k]$ code to a $[n+1, k+1]$ code to produce best equivocation codes. We present examples for the code where the number of parity bits of the code is equal to $15(m=15)$ where $m=n-k$. The code construction method for obtaining good equivocation codes is based on the observation that the syndrome probability mass function of a code extended in length is a function of the probability mass function of the original code, and good equivocation codes produce good extended codes. The design results for $m=15$ show that these new best equivocation codes (BEC) have better equivocation rate compared to all previously published best error correcting codes, the best known codes (BKC) listed by Grassl [9].

\section{SYNDROME CODING SCHEME}

Wyner showed that the secrecy capacity of the wiretap channel [1] is :

$$
C_{s}=-p_{e} \cdot \log _{2}\left(p_{e}\right)-\left(1-p_{e}\right) \cdot \log _{2}\left(1-p_{e}\right)
$$

which is the highest transmission rate that can be obtained while maintaining perfect secrecy. In this model, Alice (transmitter) wants to transmit a sequence of independent and uniformly distributed $m$-bit binary messages to Bob (legitimate receiver), $M[1], \ldots M[r]$. This sequence of messages is encoded into n-bit words $C[1], \ldots C[r]$. Bob receives the same sequence of $n$-bit words $C[1], \ldots C[r]$ and Eve receives the sequence of $n$-bit words $D[1], \ldots D[r]$ where 


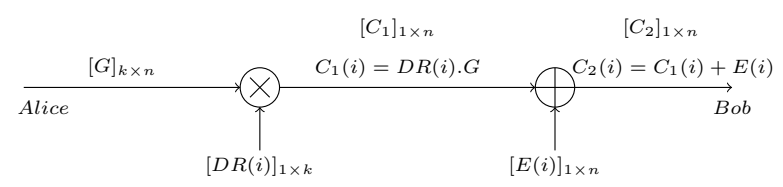

Fig. 2: Block Diagram of Syndrome Coding scheme for $(n, k, d)$ linear block code

$$
D(i)=C(i)+E_{B S C}(i), \quad i=1, \ldots, r
$$

and $E_{B S C}(i)$ represents a $n$-bit error vector generated by the binary symmetric channel, $r$ is the block length. The syndrome coding scheme uses a $(n, k, d)$ linear block code which guarantees to correct all error patterns of weight $t$, where $t=\lfloor(d-1) / 2\rfloor$. All $2^{m}$ syndromes are used to send messages where $m=n-k$ and not just the syndromes corresponding to weight $t$ or less error patterns. For any linear block code there exist $2^{m}$ distinct minimum weight error patterns, the coset leaders, in which each pattern produces a distinct syndrome of the total $2^{m}$ syndromes. Therefore, these error patterns can be represented in a table of $2^{m}$ syndromes. In the traditional syndrome coding, the look up table for error patterns and syndromes is known by Alice, Bob and Eve.

For long codes a syndrome table is impractical, but it is shown below that this look up table is unnecessary, and that the parity check matrix $H$ of the code is sufficient taking into consideration the structure of $H$ in systematic format. A block diagram for syndrome coding for a $(n, k, d)$ linear block code is shown in Fig. 2.

\section{A. Encoding Algorithm}

Alice starts the encryption process in order to generate a $n$-bit vector $C_{2}(i)$ from each $m$-bit message $M(i)$ at time $i$ such that $C_{2}(i) \times H^{T}=M(i)$ as shown in Algorithm 1 .

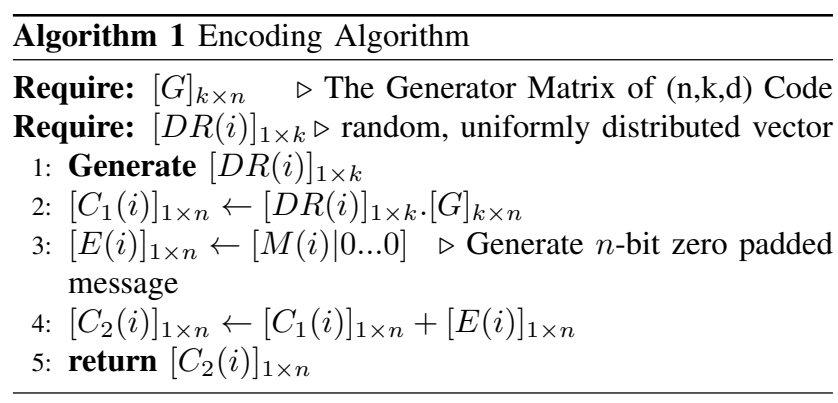

Now, we show how to calculate the error pattern $[E(i)]_{1 \times n}$. Since the syndrome of any codeword is zero, any codeword added to an error pattern will produce the same syndrome. Hence Alice may produce the required syndrome by generating an $n$-bit zero padded message vector $E(i)$, which consists of the original message $M(i)$ which is $m$-bits long followed by $k 0$ 's where $m=n-k$.

\section{B. Decoding Algorithm}

1) Legitimate Receiver's Decoder: Bob receives the transmitted vector $\left[C_{2}(i)\right]_{1 \times n}$ via the main channel that is error-

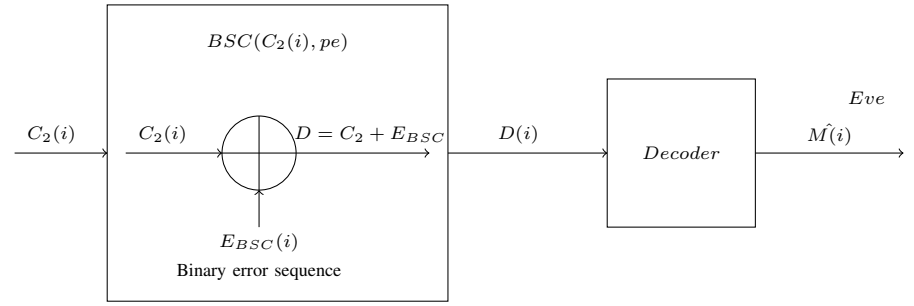

Fig. 3: Block Diagram of the BSC channel and Eavesdropper's Decoder

free. He recovers the original message $M(i)$ by using the parity check matrix of the code as shown in Algorithm 2.

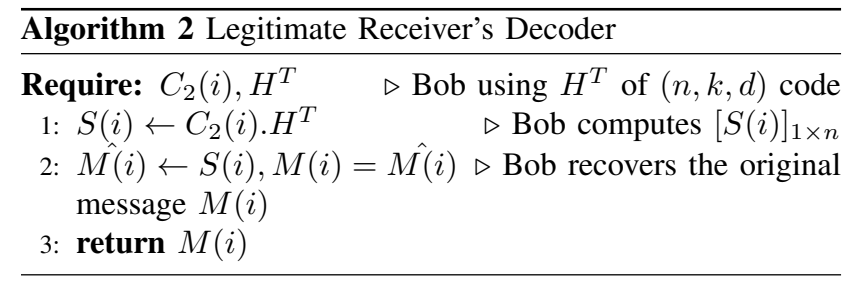

From Algorithm 2, we can prove that $C_{2}(i) \times H^{T}=M(i)$ as follows:

$S(i)=C_{2}(i) \times H^{T} \longrightarrow S(i)=\left(C_{1}(i)+E(i)\right) \times H^{T}$

$S(i)=C_{1}(i) \times H^{T}+E(i) \times H^{T}$

$S(i)=D R(i) \times G \times H^{T}+E(i) \times H^{T}$, but $G \cdot H^{T}=0$.

So, $S(i)=E(i) \times H^{T}=M(i)$.

The syndrome formed from $E(i) \times H^{T}$, because of the $k$ leading zeros of $E(i)$ is simply $M(i)$ multiplied by the identity sub-matrix of $H^{T}$ which produces $M(i)$.

2) Eavesdropper's Decoder: The block diagram of the BSC channel and eavesdropper's decoder is shown in Fig. 3. Eve receives a corrupted vector $D(i)$ instead of the transmitted vector $\left[C_{2}(i)\right]$ as a result of passing through the BSC which adds additional errors $\left[E_{B S C}\right]_{1 \times n}$ :

$[D(i)]_{1 \times n}=\left[C_{2}(i)\right]_{1 \times n}+\left[E_{B S C}(i)\right]_{1 \times n}$, Where $\left[E_{B S C}(i)\right]_{1 \times n}$ is a random binary error vector which depends on the crossover probability pe of BSC. Assuming Eve uses the same type of decoder that has been used by Bob, the following steps explains how she gets the estimated message $\hat{M(}(i)$ from the corrupted vector $D(i)$ :

\begin{tabular}{l}
\hline Algorithm 3 Eavesdropper's Decoder \\
\hline Require: $D(i), H^{T} \quad \triangleright$ Eve using $H^{T}$ of $(n, k, d)$ code \\
1: $S_{E v e}(i) \leftarrow D(i) . H^{T} \quad \triangleright$ Eve computes $\left[S_{E v e}(i)\right]_{1 \times n}$ \\
2: $M(i) \leftarrow S_{E v e}(i), M(i) \neq \hat{M(}(i) \quad \triangleright$ Eve recovers an \\
$\quad$ estimate of the message $\hat{M(}(i)$ \\
3: return $\hat{M(}(i)$ \\
\hline
\end{tabular}


From Algorithm 3, Eve estimates $\hat{M(}(i)$ as follows:

$S_{\text {Eve }}(i)=D(i) \times H^{T}$

$S_{\text {Eve }}(i)=\left[C_{2}(i)+E_{B S C}(i)\right] \times H^{T}$

$S_{E v e}(i)=C_{2}(i) \times H^{T}+E_{B S C}(i) \times H^{T}$

$S_{E v e}(i)=\left[C_{1}(i)+E(i)\right] \times H^{T}+E_{B S C}(i) \times H^{T}$

$S_{E v e}(i)=E(i) \times H^{T}+E_{B S C}(i) \times H^{T}=\hat{M(i)}$

$\hat{M(i)}=S_{E v e}(i)=M(i)+S_{e}(i)$

\section{CAlCulation OF THE SECRECy ACHIEVED BY} SYNDROME CODING

The secrecy realised by syndrome coding is measured by the eavesdropper decoder output equivocation, $H(M(i) \mid \hat{M}(i))$ :

$$
\begin{aligned}
H(M(i) \mid \hat{M}(i))= & H(M(i), \hat{M}(i))-H(\hat{M}(i)) \\
= & H(M(i))-H(\hat{M}(i))+H(\hat{M}(i) \mid M(i)) \\
= & H(M(i))-H\left(M(i)+S_{e}(i)\right)+ \\
& H\left(M(i)+S_{e}(i) \mid M(i)\right) \\
= & m-m+0+H\left(S_{e}(i) \mid M(i)\right) \\
= & H\left(S_{e}(i)\right) \\
& 2^{m}-1 \\
H(M(i) \mid \hat{M}(i))= & -\sum_{i=0} p\left(S_{e}(i)\right) \cdot \log _{2} p\left(S_{e}(i)\right)
\end{aligned}
$$

where $H\left(S_{e}(i)\right)$ is the entropy of $S e(i)$. The simplifications in equations (2) and (3) are due to $M(i)$ being uniformly distributed and independent of $S_{e}(i)$. The equivocation is calculated after deriving the probability mass function of the syndromes due to errors from the BSC, $p\left(S_{e}(i)\right)$ and is a function of the code being used through the parity check matrix of the code.

\section{A. Code Representation}

Any binary linear $(n, k, d)$ code is defined by its $(k \times n)$ generator matrix $G$ or by its $(m \times n)$ parity check matrix $H$. The best equivocation codes are constructed by designing the parity check matrix of the code. $H$ can be defined by representing each column of $H$ by an integer, $b_{i}$, in the range 0 to $\left(2^{n-k}-1\right)$. The binary parity check matrix of a code of length $n$ is defined by $n . k$ binary integers, the first $m$ columns of $H$ is an identity matrix if $H$ is in systematic form, as shown below:

$$
\mathbf{H}=\left[\begin{array}{ccccccc}
1 & 0 & \ldots & 0 & a_{m 0} & \ldots & a_{(n-1) 0} \\
0 & 1 & \ldots & 0 & a_{m 1} & \ldots & a_{(n-1) 1} \\
\vdots & \vdots & \ldots & \vdots & \vdots & a_{i j} & \vdots \\
0 & 0 & \ldots & 1 & a_{m(m-1)} & \ldots & a_{(n-1)(m-1)}
\end{array}\right]
$$

in which $0 \leq j \leq m-1, m \leq i \leq n-1$ and $a_{i, j}$ takes a value of 0 or 1 . Each column can be represented as a packed integer defined as $b_{i}=\sum_{j=0}^{m-1} a_{i j} \cdot 2^{j}$. Then the systematic format of $H$ can be represented as following:

$$
H=\left[1,2, \ldots, 2^{m-1}, b_{m}, \ldots, b_{n-1}\right]
$$

where the first $m$ integers represent the identity matrix and the other integers have values between 3 and $2^{m}-1$. Usually no integers are repeated ensuring $d \geq 3$ and higher values of $d$ are ensured by constraining no integer is a modulo 2 sum of any other $d-2$, or smaller, number of integers.

\section{B. Evaluation of the syndrome probability distribution}

The code construction technique that produces codes with good equivocation is based on the realisation that the syndrome probability mass function ( $\mathrm{pmf}$ ) of a new extended code is a function of the probability mass function of the original code and good equivocation codes produce good extended codes. For Eve, there are $2^{n}$ possible error patterns, e(i), occur for each transmitted vector $C(i)_{1 \times n}$. These error patterns occur with probability:

$$
p(e(i))=p e^{w(i)} \cdot(1-p e)^{n-w(i)}
$$

where $w(i)$ is the weight of $e(i)$. Each error pattern results in one of the $2^{m}$ syndromes being produced.

$$
\left.S_{e}(i)\right)=e(i) \times H^{T}
$$

As the code is linear, for each syndrome there are $2^{k}$ error patterns that produce the same syndrome and the probability of each syndrome due to all possible error patterns is given by:

$$
p\left(S_{j}\right)=\sum_{i=0}^{2^{n}-1} p(e(i)) . \delta\left(S_{e}(i)-S_{j}\right)
$$

where $\delta()$ is the Dirac function and

$$
H\left(S_{e}(i)\right)=-\sum_{j=0}^{2^{m}-1} p\left(S_{j}\right) \cdot \log _{2}\left[p\left(S_{j}\right)\right]
$$

This method for evaluating the equivocation works well for short codes $(n<40)$, but for the long codes it is impracticable because it involves the evaluation of $2^{n}$ error patterns. Due to the limitation of this method, the probability distribution of the syndromes may be determined recursively, this method leads to reduce the number of terms from $2^{n}$ to $2^{m}$ as shown in the following theorem.

Theorem : The probability mass function (pmf) of $S_{j}$ for $j=0$ to $2^{m}-1$ can be defined as $p\left(S_{j}\right)=\beta(j)$ where $\beta(j)$ are coefficients of the probability generating function using the $\mathrm{Z}$ transform, denoted as $p_{z}(S)$ and $p_{z}(S)$ only depends on the columns of the parity check matrix $H$ and the probability error of the binary symmetric channel $p_{e}$.

$$
p_{z}(S)=\sum_{j=0}^{2^{m}-1} \beta(j) Z^{j}=\prod_{i=0}^{n-1}\left((1-p e)+p e \cdot Z^{b_{i}}\right)
$$

where $b_{i}$ are the integers representations of the columns of $H$ and exponent sums of powers of $Z$ are added modulo 2 . Proof: Any error pattern may be represented as a sum of single bit error events: $e(i)=\left[\begin{array}{llll}e_{1} & e_{2} & \ldots & e_{n}\end{array}\right]$

$e(i)=\left[\begin{array}{llll}e_{1} & 0 & \ldots & 0\end{array}\right]+\left[\begin{array}{llll}0 & e_{2} & \ldots & 0\end{array}\right]+\cdots+\left[\begin{array}{llll}0 & 0 & \ldots & e_{n}\end{array}\right]$ where $e_{i}=1$ with probability $p_{e}$ and $e_{i}=0$ with probability $1-$ $p_{e}$. The linearity of the syndrome coding scheme means that the syndrome resulting from any error pattern is the linear sum of the syndromes for each bit error position:

$$
\begin{gathered}
S_{e}(i)=e(i) \times H^{T}=\left[\begin{array}{llll}
e_{1} & e_{2} & \ldots & e_{n}
\end{array}\right] \times H^{T} \\
S_{e}(i)=b_{1} \delta\left(e_{1}-1\right) \oplus b_{2} \delta\left(e_{2}-1\right) \cdots \oplus b_{n} \delta\left(e_{n}-1\right)
\end{gathered}
$$


The steps of the algorithm can be simplified as follows:

1) Calculate the syndrome pmf of the original code $(n, k)$ from equation(14).

2) Represent the parity check matrix $H$ of the $(n, k)$ code in the systematic format:

$$
H=\left[1,2,4, \ldots, 2^{m-1}, b_{m}, \ldots, b_{n-1}\right]
$$

3) Extend $H$ with one integer $\left(b_{r}\right)$ by generating randomly all possible integers between 3 and $2^{m}-1$ with the constraint that there are no repeated integers included in the original $H$. This ensures that the minimum Hamming distance of each extended code is at least 3 .

4) Eliminate all equivalent codes and evaluate the equivocation rate for each remaining code by using equation(4).

5) Rank the inequivalent codes by their equivocation rate in descending order, and select a best codes subset. These codes are used as the initial input for the next extension round.

\section{RESULTS}

By using the code design technique above, the best equivocation codes have been determined for $m=15$. As a result of the large number of codes we only present here in Table 1 codes which provide at least $80 \%$ secrecy. The minimum Hamming distance $(d)$ and the equivocation rate (Eq.) for a BSC error probability of $p_{e}=0.05$ is given for each code. The equivocation rates of the corresponding best error correcting codes previously published, the (BKC) codes listed by Grassl [9] with the same $\mathrm{n}$ and $\mathrm{m}$ are also given in Table 1 (in parentheses). The results show that significant improvements have been achieved on the equivocation rate for the best equivocation codes compared with best known codes.

Fig. 4 shows the equivocation rate $E q$. as a function of probability of error $p_{e}$ of best equivocation and best known codes for $n=82$ at different values of $p_{e}$. It shows that the equivocation rate of BECs has been increased by a large margin compared with $\mathrm{BKCs}$ not only for $p_{e}=0.05$ but also for other values of $p_{e}$.

\section{CONCLUSIONS}

In this paper, we presented a code design technique for obtaining best equivocation codes and also presented a method of implementing syndrome coding without the need for a syndrome look up table. The best equivocation codes for the syndrome coding scheme that achieve at least $80 \%$ secrecy to an eavesdropper using the BSC with an error probability of 0.05 are presented in Table 1 . In addition, a recursive method for the evaluation of the probability mass function of the syndromes of a code which depends only on the columns of the parity check matrix and the probability of error of the binary symmetric channel has been presented. The results obtained show that the equivocation rate of the new best equivocation codes exceeds by a large margin the equivocation rates of the best error correcting codes, previously published.

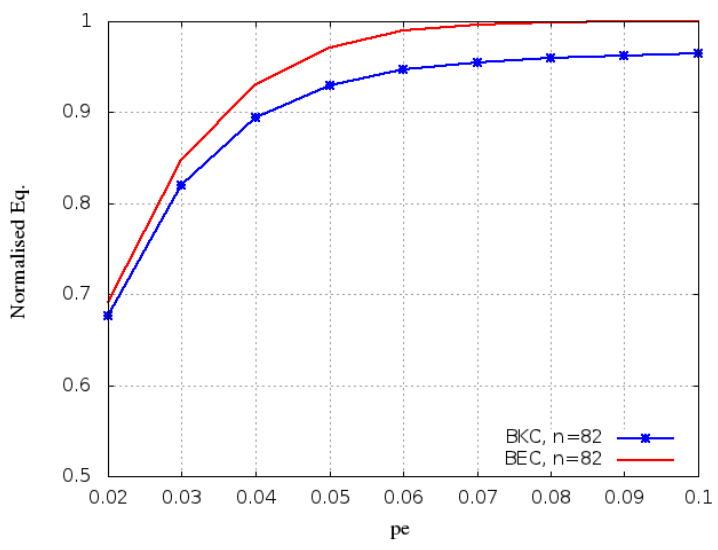

Fig. 4: Equivocation rate $E q$. vs. $p_{e}$ of best equivocation (BEC) and best known (BKC) codes for $n=82$

\section{REFERENCES}

[1] A. D. Wyner, "The Wire-Tap Channel," The Bell System Technical Journal, vol. 54, pp. 1355-1367, May 1975.

[2] L. H. Ozarow and A. D. Wyner, "Wire-tap channel ii," The Bell System Technical Journal, vol. 63, no. 10, pp. 2135-2157, 1984.

[3] Y. Chen and A. J. Vinck, "On the binary symmetric wiretap channel," Int. Zurich Seminar on Communications(IZS), pp. 17-20, 3-5 March 2010.

[4] S. Y. E. Rouayheb and E. Soljanin, "On wiretap networks ii," ISIT, pp. 551-555, 24-29 June 2007.

[5] S. Al-Hassan, M. Ahmed, and M. Tomlinson, "Secrecy coding for the wiretap channel using best known linear codes," in Global Information Infrastructure Symposium, 2013, Oct 2013, pp. 1-6.

[6] G. Cohen and G. Zemor, "Syndrome-coding for the wiretap channel revisited," in Information Theory Workshop, 2006. ITW '06 Chengdu. IEEE, 2006, pp. 33-36.

[7] W. Alltop, "A method for extending binary linear codes (corresp.)," Information Theory, IEEE Transactions on, vol. 30, no. 6, pp. 871-872, Nov 1984.

[8] Y. Edel and J. Bierbrauer, "Inverting construction y1," Information Theory, IEEE Transactions on, vol. 44, no. 5, pp. 1993-, Sep 1998.

[9] M. Grassle, "Bounds on the minimum distance of linear codes and quantum codes," 2007,online, Available:http://www.codetables.de. 
TABLE I: Best Equivocation Codes that achieve at least $80 \%$ secrecy in syndrome coding for $p_{e}=0.05$

\begin{tabular}{|c|c|c|c|c|}
\hline $\mathrm{m}$ & $\mathrm{n}$ & d & Eq. & Packed integer parity check matrix \\
\hline 15 & 48 & 5 & $\begin{array}{ll}0.804141 \\
(0.744035)\end{array}$ & $\begin{array}{l}124816326412612825651282610242048387940967163791381929215963210552163841697517378177791884319664211362197322578233932409224495 \\
2514426321264092664026663274112809228622293022997731397318713239532607\end{array}$ \\
\hline 15 & 49 & 5 & $\begin{array}{l}0.813668 \\
(0.772691)\end{array}$ & $\begin{array}{l}124816326412825651210242048387940965541703171607913819292151398714289163841697517378 \\
23393240922449525609256982632126409274112809228133286222881631397316753187132153\end{array}$ \\
\hline 15 & 50 & 5 & $\begin{array}{l}0.822836 \\
(0.780014)\end{array}$ & 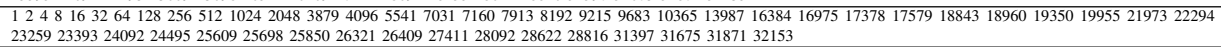 \\
\hline 15 & 51 & 5 & $\begin{array}{l}0.831624 \\
(0.787048)\end{array}$ & 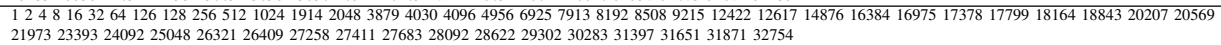 \\
\hline 15 & 52 & 5 & $\begin{array}{l}0.840045 \\
(0.793774)\end{array}$ & 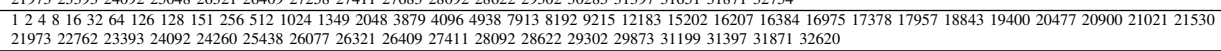 \\
\hline 15 & 53 & 5 & $\begin{array}{l}0.848118 \\
(0.800219)\end{array}$ & $\begin{array}{l}1248163264128256512102420483879409647975541703171607913819292151398714289143711638416975173781757918180182931884318960 \\
199552197322537232592339324092244952560925698263212640927411280922813328622 \\
18881631397316753187132153\end{array}$ \\
\hline 15 & 54 & 5 & $\begin{array}{l}0.855849 \\
(0.806378)\end{array}$ & $\begin{array}{l}124816326412825651210242048369138794096554163157031716074897913819292151398714289163841665316975173781757918843189601935019955 \\
2197323259233932409224495256092569826321264092741127517280922813328622288163071731397316753187132153\end{array}$ \\
\hline 15 & 55 & 5 & $\begin{array}{l}0.863253 \\
(0.812037)\end{array}$ & 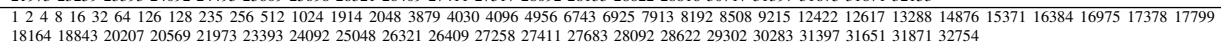 \\
\hline 15 & 56 & 5 & $\begin{array}{l}0.870337 \\
(0.817515)\end{array}$ & 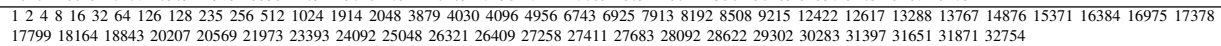 \\
\hline 15 & 57 & 5 & $\begin{array}{l}0.877108 \\
(0.822674)\end{array}$ & 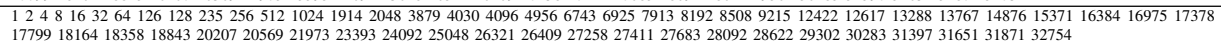 \\
\hline 15 & 5 & 5 & $\begin{array}{l}0.883542 \\
(0.827531)\end{array}$ & 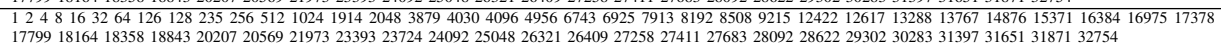 \\
\hline 15 & 59 & 5 & $\begin{array}{l}0.889691 \\
(0.832243)\end{array}$ & 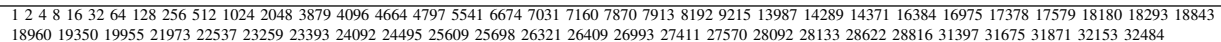 \\
\hline 15 & 60 & 5 & $\begin{array}{l}0.895581 \\
(0.836659)\end{array}$ & 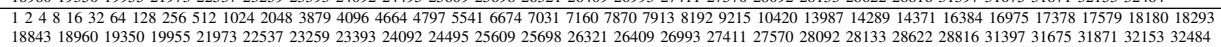 \\
\hline 15 & 61 & 5 & $\begin{array}{l}0.901181 \\
(0.84071)\end{array}$ & 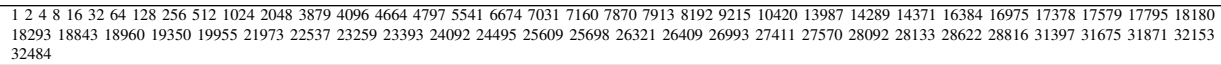 \\
\hline 15 & 62 & 5 & $\begin{array}{l}0.906529 \\
(0.84471)\end{array}$ & $\begin{array}{l}12481632641261282352565121024191420483879403040964956674369257913819285089215106381242212617132881376714876153711638416975 \\
1737817758177991816418358188432020720569219732339323724238552409225048263212640927258274112768328092286222916329302302833139731651 \\
3187132754\end{array}$ \\
\hline 15 & 63 & 5 & $\begin{array}{l}0.911620 \\
(0.848502)\end{array}$ & $\begin{array}{l}124816326412612825651210241914204831453879403040964956536862566454692579138192850892151062711106124221261714876152541638416604 \\
1697517378177991816418324187001884320207205692197322743233932409225048263212640927258274112768328092286222930230283309613139731651 \\
3187132754\end{array}$ \\
\hline 15 & 64 & 5 & $\begin{array}{l}0.916471 \\
(0.852014)\end{array}$ & $\begin{array}{l}1248163264126128256512102419142048314538793940403040964956535953686256645469257913819285089215106271110612422126171487615254 \\
163841660416975173781779918164183241884320207205692197322743233932409225048263212640927258274112768328092286222930230283 \\
316513187132754\end{array}$ \\
\hline 15 & 65 & 5 & & 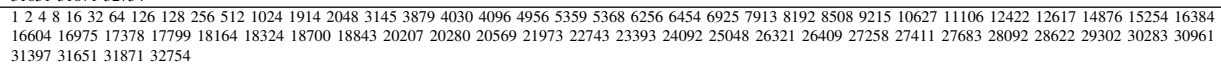 \\
\hline 15 & 66 & 5 & $\begin{array}{l}0.925470 \\
(0.858469)\end{array}$ & $\begin{array}{l}124816326412612825651210241914204831453879394040304096495653686256645465646925791381928508921592641062711106124221261714876 \\
152541638416604169751737817799181641832418843202072056921973227432339324092250482514826321264092725827411276832809228622 \\
3096131397316513187132754\end{array}$ \\
\hline 15 & 67 & 5 & & $\begin{array}{l}124816326412612825651210241914204831453879394040304096495653686256645465646925791381928508921592641062711106124221261714876 \\
152541638416604169751737817799181641832418843202072056921522219732274323393240922504825148263212640927258274112768328092 \\
302862233096131397316513187132754\end{array}$ \\
\hline 15 & 68 & 5 & $\begin{array}{l}\begin{array}{l}0.933609 \\
(0.873716)\end{array}\end{array}$ & 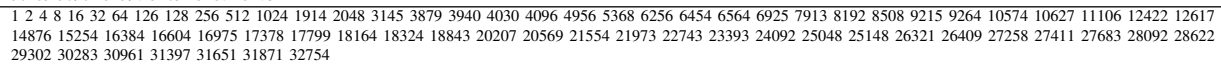 \\
\hline 15 & 69 & 5 & $\begin{array}{l}0.937373 \\
(0.883069)\end{array}$ & $\begin{array}{l}124816326412612825651210241914204831453879394040304096495653686256645465646925791381928508921592641062711106124221261714876 \\
152541638416604169751737817602177991816418324188432020720569215222197322743233932409225048251482632126409272112725827411 \\
286222968302302833096131397316513187132754\end{array}$ \\
\hline 15 & 70 & 5 & & 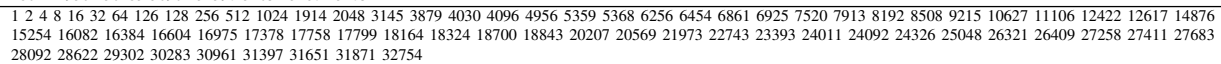 \\
\hline 15 & 71 & 5 & $\begin{array}{l}0.944327 \\
(0.896977)\end{array}$ & $\begin{array}{l}124816326412612825651210241914204831453879403040964956535953686256645468616925752079138192850892151062711106124221261713924 \\
148761525416082163841660416975173781775817799 \\
27683280922862229302302833096131397316513187132754\end{array}$ \\
\hline 15 & 72 & 5 & $\begin{array}{l}0.947533 \\
(0.902252)\end{array}$ & $\begin{array}{l}124881632641261282565121024191420482697314558794030409649565359536862566454686169257520791381928508921510627111061242212617 \\
1487615254160821638416604169751737817758177991816418324187001884320207205692197322743233932401124092243262504826321264092725827411 \\
2768328092286222930230283309613139731651318713200732754\end{array}$ \\
\hline 15 & 73 & 5 & & 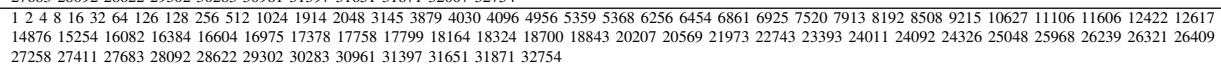 \\
\hline 15 & 74 & 5 & $\begin{array}{l}0.953458 \\
(0.910715)\end{array}$ & 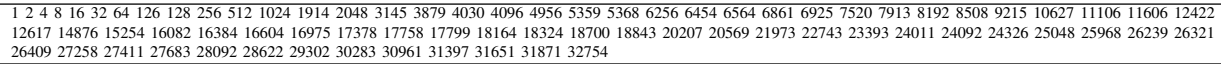 \\
\hline 15 & 75 & 5 & & $\begin{array}{l}12481632641261282565121024191420483145387940304096495653595368625664546564686169257520791381928508921510627111061160612422 \\
1261714876152541608216384166041697517378177581779918164183241870018843202072056921973227432339324011240922432625048259682623926321 \\
2640927258274112768328092286222930230283309613139731651318713213332754\end{array}$ \\
\hline 15 & 76 & 5 & & 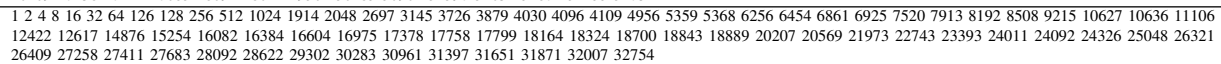 \\
\hline 15 & 77 & 4 & $\begin{array}{l}0.961205 \\
(0.919134)\end{array}$ & 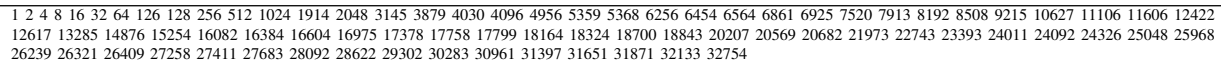 \\
\hline 15 & 78 & 5 & & 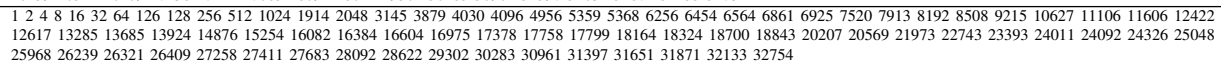 \\
\hline 15 & 79 & 5 & & 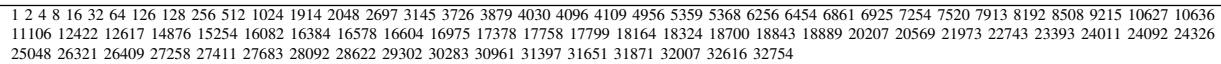 \\
\hline 15 & 80 & 5 & $\begin{array}{l}0.967762 \\
(0.926307)\end{array}$ & 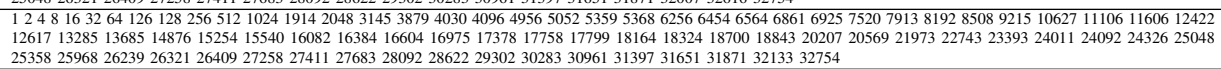 \\
\hline 15 & 81 & 5 & & 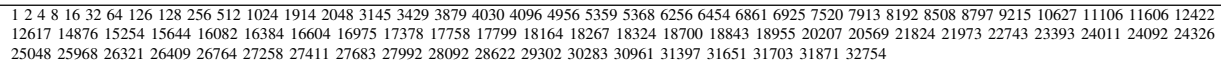 \\
\hline 15 & 82 & 5 & & $\begin{array}{l}124816326412612825651210241914204831453429387940304096495653595368625664546861692575207913819285088797921510627111061160612422 \\
1261714876152541564416082163841660416975173781775817799181641826718324187001884318955202072056921824219732274323393240112409224326 \\
2504825968263212640926764272582741127683279922809228622293023028330961313973165131703318713270932754\end{array}$ \\
\hline
\end{tabular}

